\title{
MRI-based three-dimensional reconstruction for staging cervical cancer and predicting high-risk patients
}

\author{
Jingjing Zhang ${ }^{1,2 \wedge}$, Yingteng Wang ${ }^{3}$, Dongyan $\mathrm{Cao}^{2 \wedge}$, Keng Shen ${ }^{2}$ \\ ${ }^{1}$ Department of Obstetrics and Gynecology, The First Affiliated Hospital, Sun Yat-sen University, Guangzhou, China; ${ }^{2}$ Department of Obstetrics \\ and Gynecology, Peking Union Medical College Hospital, Chinese Academy of Medical Science \& Peking Union Medical College, National Clinical \\ Research Center for Obstetric \& Gynecologic Diseases, Beijing, China; ${ }^{3}$ Department of Radiology, Huazhong University of Science and Technology \\ Union Shenzhen Hospital (Nanshan Hospital), Shenzhen, China \\ Contributions: (I) Conception and design: K Shen, D Cao; (II) Administrative support: K Shen; (III) Provision of study materials or patients: D Cao; (IV) \\ Collection and assembly of data: J Zhang; (V) Data analysis and interpretation: J Zhang; (VI) Manuscript writing: All authors; (VII) Final approval of \\ manuscript: All authors. \\ Correspondence to: Professor Keng Shen. Department of Obstetrics and Gynecology, Peking Union Medical College Hospital, Chinese Academy of \\ Medical Science \& Peking Union Medical College, National Clinical Research Center for Obstetric \& Gynecologic Diseases, No. 1 Shuaifuyuan, \\ Dongcheng District, Beijing 100730, China. Email: shenkengkk@163.com.
}

Background: Cervical tumors usually have an irregular morphology. It is often difficult to estimate tumor size or volume based on a diameter measurement from a two-dimensional magnetic resonance imaging slice. This study aimed to explore the use of magnetic resonance imaging-based three-dimensional reconstruction in cervical cancer.

Methods: We retrospectively created a three-dimensional reconstruction based on the pre-treatment magnetic resonance imaging data of 54 cervical cancer patients at a single center to evaluate tumor size and extent of invasion, as well as to review cervical cancer staging and treatment. The tissues and organs were automatically outlined by the three-dimensional application, based on the signal intensity difference of magnetic resonance imaging data.

Results: The maximum tumor diameters calculated using the magnetic resonance imaging-based threedimensional reconstruction were larger than those calculated from the direct magnetic resonance imaging findings or gynecological examinations. Initial underestimation of the maximum tumor diameter led to under-staging in up to $29.6 \%$ of patients. The magnetic resonance imaging-based three-dimensional reconstruction revealed that upstaging was warranted based on lymph node metastasis (3.7\% of patients) and invasion of the vaginal fornix (1.9\% of patients). Lymph node metastasis was associated with a significantly larger tumor volume $(\mathrm{P}<0.05)$. A volume cut-off value $\geq 18.6 \mathrm{~mL}$ provided $60 \%$ sensitivity, $96.7 \%$ specificity, $75 \%$ positive predictive value and $93.5 \%$ negative predictive value for predicting high-risk patients $(\mathrm{P}<0.05)$.

Conclusions: Magnetic resonance imaging-based three-dimensional reconstruction is a new approach that could potentially measure cervical cancer more accurately.

Keywords: Cervical cancer; three-dimensional reconstruction; magnetic resonance imaging; tumor volume; lymph node metastasis

Submitted May 05, 2021. Accepted for publication Aug 16, 2021.

doi: 10.21037/atm-21-2246

View this article at: https://dx.doi.org/10.21037/atm-21-2246

^ ORCID: Jingjing Zhang, 0000-0002-0169-7124; Dongyan Cao, 0000-0003-4253-832X. 


\section{Introduction}

Cervical cancer is the fourth most common cancer among women worldwide (1). The International Federation of Gynecology and Obstetrics (FIGO) staging system for cervix uteri is a clinical staging system, wherein the accurate measurement of cervical cancer lesion size plays an important role. The 2018 FIGO staging system for cervix uteri added a tumor diameter of $\geq 2 \mathrm{~cm}$ as the new threshold for stage IB2 disease, and a tumor diameter of $\geq 4 \mathrm{~cm}$ was used to define stage IB3 disease (2). However, cervical tumors usually have an irregular three-dimensional morphology with many concavities and variable locations on the cervix, and approximately $55 \%$ of cervical tumors have a non-ellipsoid configuration (3). Magnetic resonance imaging (MRI) is recognized as a preferred option used to examine the cervical cancer lesions (4). However, this method can only produce a rough estimate of cervical cancer size based on a diameter measurement from a two-dimensional MRI slice. Because it can offer observation from multiple angles, the MRI-based three-dimensional (MRI3D) reconstruction may serve as a new approach to accurately measure cervical cancer lesion size.

Furthermore, stage III cervical cancer was classified as IIIC (p) and IIIC (r) in the 2018 FIGO staging system, which increases the importance of predicting lymph node metastasis (LNM). LNM is one of the adverse pathological factors of cervical cancer. Postoperative patients are categorized into low-, intermediate- or high-risk according to these factors. Adjuvant chemoradiotherapy (CRT) was recommended to intermediate- and high-risk patients (2). Furthermore, initial CRT could be considered as an option for cervical cancer patients who were at risk of adjuvant CRT to avoid surgery and its related complications. Additionally, the automatic calculation of cervical cancer volume by MRI-3D reconstruction is more accurate, timesaving, and labor-saving than that produced by MRI.

The present study aimed to (I) evaluate whether MRI-3D reconstruction could assist cervical cancer staging, and (II) explore the value of automatically calculated tumor volume by MRI-3D in predicting adverse pathological factors in intermediate- or high-risk patients. We present the following article in accordance with the STARD reporting checklist (available at https://dx.doi.org/10.21037/atm-21-2246).

\section{Methods}

\section{Patients}

This was a retrospective study. We consecutively enrolled
54 pathology-confirmed cervical cancer patients aged 18 years or older who underwent MRI within 4 weeks before treatment between September 2019 and December 2019 at the gynecological oncology department, Peking Union Medical College Hospital. Staging was determined based on the 2018 cervical cancer FIGO stage. We used findings from pelvic MRI, chest radiography, urinary system ultrasonography, and direct gynecological examinations. Re-staging defined as a reviewed stage was determined according to findings from MRI-3D reconstruction. The study was conducted in accordance with the Declaration of Helsinki (as revised in 2013). Ethical approval was granted by the institutional review board of Peking Union Medical College Hospital (approval number: JS-2082). Written informed consent for participation was waived for this retrospective study.

\section{MRI Protocol and 3D reconstruction}

Pelvic MRI was performed using an Ingenia Elition 3.0T scanner (Philips Company) with a dedicated pelvic coil. Oral administration of $10 \mathrm{mg}$ raceanisodamine was prescribed 30 minutes before the MRI examination to minimize bowel-related motion artifacts. The MRI protocol included the following sequences: T1-weighted [T1W; repetition time (TR), $450 \mathrm{~ms}$; echo time (TE), $12.1 \mathrm{~ms}$; field of view (FOV), $280 \mathrm{~mm} \times 280 \mathrm{~mm}$; matrix size, $256 \times 224$; slice thickness/interval, $5 / 1.5 \mathrm{~mm}$; number of excitations (NEX), 4], gadolinium contrast-enhanced, T1-weighted images with fat suppression (CET1W; TR, $1.8 \mathrm{~ms}$; TE, $3.8 \mathrm{~ms}$; FOV, $280 \mathrm{~mm} \times 280 \mathrm{~mm}$; matrix size, 256×192; slice thickness/interval, 5/1.5 mm; NEX, 1), T2-weighted (T2W; TR, $4300 \mathrm{~s}$; TE, $95 \mathrm{~ms}$; FOV, $180 \mathrm{~mm} \times 80 \mathrm{~mm}$; matrix size, 256×224; slice thickness/interval, 5/1.5 mm; NEX, 2), and 3D-SPACE-STIR (TR, $1700 \mathrm{~ms}$; TE, $102 \mathrm{~ms}$; FOV, $250 \mathrm{~mm} \times 250 \mathrm{~mm}$; matrix size, $320 \times 272$; slice thickness/ interval, 1/0 mm; NEX, 2). A contrast agent was intravenously injected into the patient (gadolinium-dextran, 20 mL, 9.38 g; Beijing Beilu Pharmaceutical Co., Ltd.) at a dose of $0.1 \mathrm{mmol} / \mathrm{kg}$.

The pelvic imaging data were saved in the Digital Imaging and Communications in Medicine (DICOM) format and emailed to VitaWorks ${ }^{\circledR}$ Technology Co., Ltd. for MRI-3D reconstructions using VitaWorks ${ }^{\circledR}$, a medical 3D Visualization Analysis System. The pelvis, iliac vessels, pelvic lymph nodes (with enlargement), bladder, urethra, rectum, uterus, cervical tumour(s), parametrial tissue (if there was thickening), and vagina were marked 

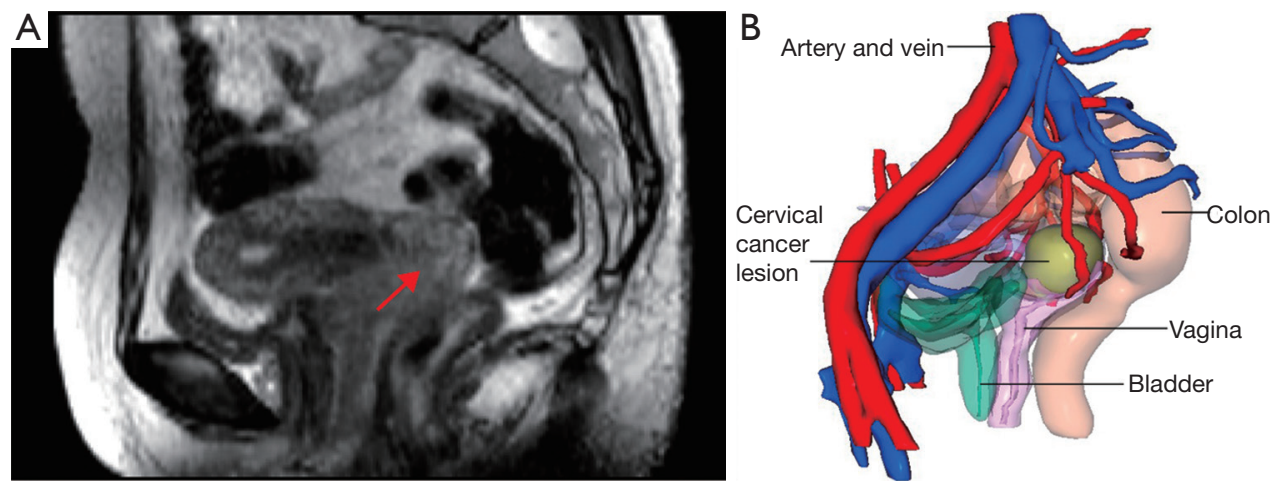

Figure 1 MRIs of a patient with cervical cancer and 3D reconstructed cervical cancer image. The boundary of tumor is automatically outlined and tumor volume was automatically calculated by VitaWorks ${ }^{\circledR}$, whose procedure based on signal intensity difference between the background and anatomical features. (A) Sagittal image, Arrow: cervical cancer lesion. (B) MRI-3D reconstructed image. MRI-3D, magnetic resonance image based three-dimensional.

with different colours (Figure 1). VitaWorks ${ }^{\circledR}$ was set to automatically recognize the signal intensity difference of 3D-SPACE-STIR imaging sequence and perform 3D reconstruction.

\section{Assessing tumor size and extent of invasion}

On 3D-MRI reconstructions, the following parameters were measured: maximum tumor diameter, tumor volume, pelvic lymph node metastasis (LNM) and/or parametrial infiltration, and morphological integrity of the bladder, rectum, and vagina. The maximum tumor diameter was measured twice by the same investigator and the mean value was used for the analysis. The cervical tumor volume was calculated automatically using VitaWorks ${ }^{\circledR}$. The presence of LNM and/or parametrial infiltration was considered as positive based on outlined structures that were identified using VitaWorks ${ }^{\circledR}$; the absence of LNM and/or parametrial infiltration outlined by VitaWorks ${ }^{\circledR}$ was considered as negative. Any loss of morphological integrity at the bladder, rectum, or vagina was interpreted as cervical cancer invasion.

\section{Postoperative pathological findings}

Adverse pathological factors include LNM, parametrial infiltration, positive margins, deep stromal infiltration, lymphovascular invasion, and tumor size $\geq 4 \mathrm{~cm}$. Patients were defined as high risk if they had any one of first three pathological adverse factors (LNM, parametrial infiltration, positive margins). Patients were defined as intermediate risk if they had any two of last three pathological adverse factors (deep stromal infiltration, lymphovascular invasion, tumor size $\geq 4 \mathrm{~cm}$ ).

\section{Statistical analysis}

The statistical analyses were performed using SPSS software (version 25.0; IBM Corp.) and differences were considered statistically significant at two-sided $\mathrm{P}$ values $<0.05$. The maximum tumor diameter measured by MRI3D reconstruction and MRI were compared using Student's $t$-test. The maximum tumor diameter measured by MRI$3 \mathrm{D}$ reconstruction and gynecological examination were compared using Student's $t$-test. The intraclass correlation coefficient (ICC) was used to evaluate the repeatability of multiple measurements of the same parameter by the same investigator. The predictive value and the cut-off threshold were calculated using the area under the receiver operating characteristic (ROC) curve.

\section{Results}

Table 1 shows the clinicopathological characteristics of the 54 patients investigated in this study. 54 patients with an average age of 49 years were enrolled. There were 41 cases of squamous cell carcinoma and 13 cases of adenocarcinoma or adenosquamous cell carcinoma. There were 49 cases of early-stage cervical cancer (stage I \& II), and 5 cases of advanced-stage cervical cancer (stage III \& IV). The treatment strategies involved giving up on treatment $(5 / 54$, $9.3 \%)$, concurrent CRT $(9 / 54,16.7 \%)$, surgery only $(24 / 54$, 
Table 1 Clinicopathological characteristics of the 54 patients in the study

\begin{tabular}{|c|c|}
\hline & $\mathrm{n}(\%)$ \\
\hline \multicolumn{2}{|l|}{ Characteristics } \\
\hline \multicolumn{2}{|l|}{ Age (years) } \\
\hline $\mathrm{M} \pm \mathrm{SD}$ & $49.0 \pm 11.5$ \\
\hline Range & $24-73$ \\
\hline \multicolumn{2}{|l|}{ Histology } \\
\hline SCC & $41(75.9)$ \\
\hline AC or ASC & $13(24.1)$ \\
\hline \multicolumn{2}{|l|}{ FIGO stage (2018) } \\
\hline IA1 & $2(3.7)$ \\
\hline IA2 & $2(3.7)$ \\
\hline IB1 & $19(35.2)$ \\
\hline IB2 & $14(25.9)$ \\
\hline IB3 & $3(5.6)$ \\
\hline$\| \mathrm{A} 1$ & $1(1.9)$ \\
\hline IIA2 & $2(3.7)$ \\
\hline IIB & $6(11.1)$ \\
\hline IIIB & $2(3.7)$ \\
\hline IIIC & $2(3.7)$ \\
\hline IVA & $1(1.9)$ \\
\hline Adverse pathological factors & n (\%) \\
\hline \multicolumn{2}{|l|}{ LNM } \\
\hline Positive & $5(13.9)$ \\
\hline Negative & $31(86.1)$ \\
\hline \multicolumn{2}{|l|}{ Parametrial infiltration } \\
\hline Positive & 0 \\
\hline Negative & $35(100.0)$ \\
\hline \multicolumn{2}{|l|}{ Positive margins } \\
\hline Positive & 0 \\
\hline Negative & $35(100.0)$ \\
\hline \multicolumn{2}{|l|}{ Deep stromal infiltration } \\
\hline Positive & $12(34.3)$ \\
\hline Negative & $23(65.7)$ \\
\hline \multicolumn{2}{|l|}{ Lymphovascular invasion } \\
\hline Positive & $10(28.6)$ \\
\hline Negative & 25 (71.4) \\
\hline \multicolumn{2}{|l|}{ Tumor size } \\
\hline$\geq 4 \mathrm{~cm}$ & $1(2.9)$ \\
\hline$<4 \mathrm{~cm}$ & $34(97.1)$ \\
\hline
\end{tabular}

$\mathrm{M} \pm \mathrm{SD}$, mean \pm standard deviation; SCC, squamous cell carcinoma; AC, adenocarcinoma; ASC, adenosquamous cell carcinoma; LNM, lymph node metastasis.
$44.4 \%)$, surgery plus adjuvant CRT (13/54, 24.1\%), and neoadjuvant CRT, surgery, and adjuvant CRT (3/54, 5.6\%). Among patients who received surgery \pm adjuvant CRT, 35 underwent hysterectomy and pelvic lymphadenectomy. According to the postoperative pathology results, were 5, 6 , and 25 patients were identified as high risk, intermediate risk, and low risk, respectively.

On the 3D-MRI reconstruction, the investigator's measurements of maximum tumor diameter were highly consistent (ICC: $0.988,95 \%$ confidence interval: 0.979 0.993, $\mathrm{P}<0.001)$. The median maximum tumor diameter was $33.0 \mathrm{~mm}$ (range: $6.4-73.9 \mathrm{~mm}$ ) and the median tumor volume was $5.5 \mathrm{~mL}$ (range: $0.1-133.5 \mathrm{~mL}$ ). The maximum tumor diameter measured using the MRI-3D reconstruction was non-significantly larger than the measurement from the direct MRI findings $(P=0.79)$, and was significantly larger than the measurement from the gynecological examination $(\mathrm{P}<0.05)$. Based on the maximum tumor diameter from the MRI-3D reconstruction, underestimation of the FIGO stage was noted for 16 patients $(16 / 54,29.6 \%)$, and 12 of these patients required upgraded hysterectomy types (Table 2).

Each patient staged IIIB and staged IB1 (2/54, 3.7\%) had LNM presented by the MRI-3D reconstruction, and these cases were upstaged to stage IIIC (r). For patients staged IIIB, this mis-staging did not influence the choice of treatment. Conversely, for patients staged IB1, the appropriate should have been concurrent CRT, rather than surgery. Furthermore, a patient staged IB3 $(1 / 54,1.9 \%)$ had lost morphological integrity at the vaginal fornix presented by the MRI-3D reconstruction, which should have been classified as stage IIA2. However, this mis-staging would not have influenced the choice of treatment.

A comparison of the groups with and without LNM revealed a significant difference in tumor volume $(\mathrm{P}<0.05)$ and a non-significant difference in maximum tumor diameter $(\mathrm{P}>0.5)$. The median tumor volumes were $2.9 \mathrm{~mL}$ (range: $0.1-21.2 \mathrm{~mL}$ ) for patients without LNM and $18.8 \mathrm{~mL}$ (range: 2.3-24.0 mL) for patients with LNM (Figure 2A). There was a significant difference in tumor volume between the low- or intermediate-risk and high-risk groups $(\mathrm{P}<0.05)$, as well as a non-significant difference in maximum tumor diameter $(\mathrm{P}>0.5)$. The median tumor volumes were $2.9 \mathrm{~mL}$ (range: $0.1-21.2 \mathrm{~mL}$ ) for the low- or intermediate-risk group and $18.8 \mathrm{~mL}$ (range: $2.3-24.0 \mathrm{~mL}$ ) for the high-risk group (Figure 2B). A larger tumor volume had predicted value for high-risk patients $(\mathrm{P}<0.05)$, with a cut-off value of $\geq 18.6 \mathrm{~mL}$ providing $60 \%$ sensitivity, $96.7 \%$ specificity, $75 \%$ positive predictive value (PPV) and $93.5 \%$ negative predictive value 
Table 2 FIGO stage (2018), reviewed stage according to MRI-3D reconstruction model and subsequent change of hysterectomy type

\begin{tabular}{llll}
\hline FIGO stage (2018) & Reviewed stage & Hysterectomy types upgrade \\
\hline IA1 & IB1 & $\begin{array}{l}\text { Subradical hysterectomy instead of extrafascial hysterectomy (If stage IA1 without } \\
\text { lymphovascular invasion) }\end{array}$ \\
IA2 & IB1 & No & Radical hysterectomy instead of subradical hysterectomy \\
IA2 & IB2 & Radical hysterectomy instead of subradical hysterectomy \\
IB1 & IB2 & Radical hysterectomy instead of subradical hysterectomy \\
IB1 & IB3 & No & 1 \\
IB2 & IB3 & 3
\end{tabular}

a, according to the MRI-3D reconstruction, the stages of the enrolled patients were reviewed. FIGO, the International Federation of Gynecology and Obstetrics; MRI-3D, magnetic resonance image based three-dimensional.
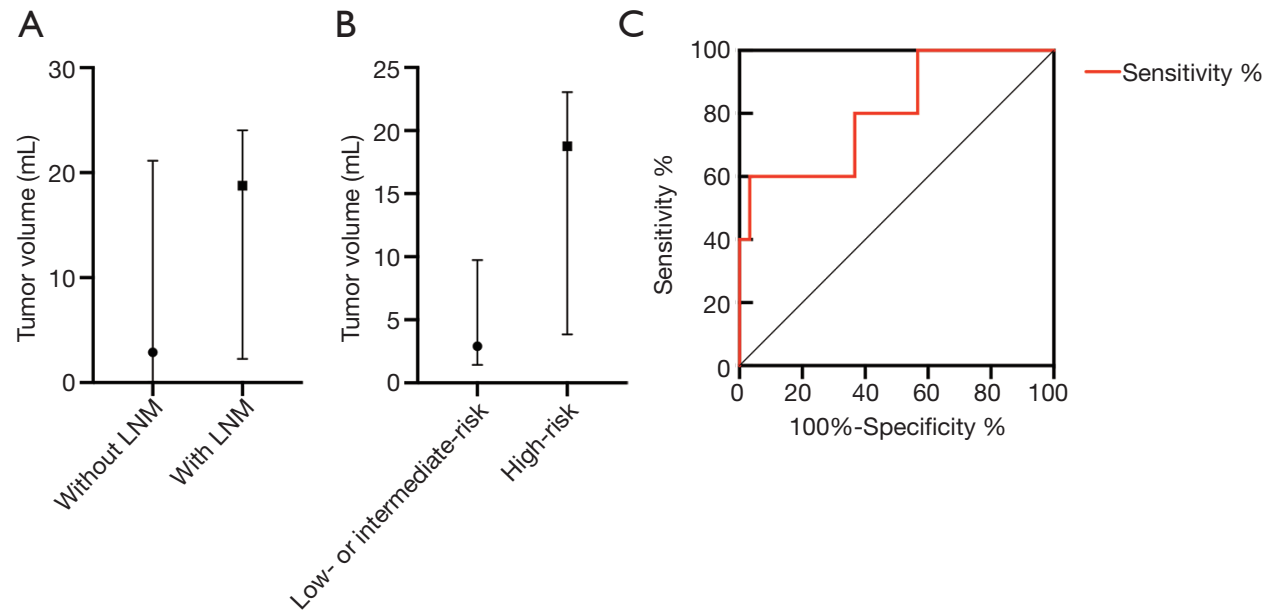

Figure 2 Tumor volume calculated by VitaWorks ${ }^{\circledR}$ in the groups with or without LNM and the groups of low- or intermediate-risk and high-risk patients and ROC curve of tumor volume in prediction of high-risk cervical cancer patients. (A) Tumor volume in the groups with or without LNM. (B) Tumor volume in the groups of low- or intermediate-risk and high-risk patients. (C) ROC curve of tumor volume in prediction of high-risk patients. LNM, lymph node metastasis; ROC, receiver operating characteristic.

(NPV) (area under the ROC curve: 0.807, 95\% confidence interval: 0.588-1.00) (Figure 2C).

\section{Discussion}

The development of new imaging modalities that provide more details on the disease could affect its diagnosis and treatment. MRI-3D reconstruction involves post-processing and secondary analysis of MRI data, which has already been applied in tumor volume calculations of oral tongue cancer and kidney cancer $(5,6)$, measuring parameters such as liver volume (7) and navigation of nerve-sparing radical hysterectomy (8).

In our study, MRI-3D reconstruction was first applied to cervical cancer staging. Up to approximately $35 \%$ of patients required upstaging because of a larger tumor size, LNM, and vaginal fornix invasion evaluated by MRI-3D reconstruction. Under-staging may lead to inadequate treatment choices including inappropriate hysterectomy types and unnecessary initial surgical treatment, which may then lead to the need for adjuvant CRT after surgery. However, initial surgery plus postoperative adjuvant therapy will make patients prone to complications (9).

We identified 2 cases of stage IA1 that were upstaged to stage IB 1 based on findings from the MRI-3D reconstruction. Given the substantial difference between a micro-invasive lesion and a grossly visible tumor, we noted that both patients underwent MRI after conization. The 
unabsorbed suture and surrounding tissue edema mimicked a cervical tumor on the MRI, and were incorporated into the MRI-3D reconstruction. The quality of the MRI$3 \mathrm{D}$ reconstruction depends on the MRI data. Specific MRI sequences are needed for different target lesions. For example, recent studies attempted to identify LNM using various MRI sequences or related methods, such as diffusion-weighted imaging (10) and dynamic contrastenhanced MRI (11). Another study implies that Zonal Oblique Multislice-diffusion weighted imaging might be useful for identifying parametrial infiltration and vaginal invasion of cervical cancer (12). The present study used a thinner slice thickness, which is not commonly used in clinical practice. Thicker slices usually produce the loss of morphological details, while the current thinner slices provide greater detail.

Measurements of maximum tumor diameter and tumor volume using MRI-3D reconstruction appear to be more convenient and accurate than direct MRI-based measurements. Specifically, measurements of maximum tumor diameter on the 3D-MRI reconstruction are not limited to the sagittal, axial, or coronal positions. Multi-angle observation helps to more accurately measure maximum tumor diameter. Furthermore, the acquisition of tumor volume data from the MRI-3D reconstruction is automatic, while manual delineation of cervical cancer tumor on direct MRI findings is time-consuming, labor-intensive and the required tumor volume estimation requires assistance of formulas or applications. Previous studies that estimate cervical tumor volume based on direct MRI findings have been constrained by these obvious disadvantages (13-16).

Chen et al. (14) and Chen et al. (17) both reported that cervical cancer tumor volume was associated with LNM. In addition, Chen et al. (14) also reported that cervical cancer tumor volume provided superior predictive value to twodimensional parameters. Our findings are consistent with Chen et al.'s and we also found that tumor volume can potentially predict high-risk patients. LNM is a high-risk adverse pathological factor of cervical cancer, indicating postoperative adjuvant CRT. A recent study showed that cervical cancer tumor volume was larger in patients with LNM than in those without LNM (16). However, this difference did not reach statistical significance. Furthermore, this study found that tumor volume was a great predictor of high-risk early cervical cancer patients.

This study has several limitations. First, selection bias may have occurred because this study took place at a tertiary referral university teaching hospital. The enrolled patients are representative of hospital-based cervical cancer patients, rather than patients from the general population. Second, the small sample size of this study reduces the reliability of our data. A larger study is needed to verify our findings and clarify the relationship between tumor volume and the adverse pathological factors of cervical cancer. The predictive value of tumor volume in cervical cancer outcome is also worth exploring.

\section{Conclusions}

MRI-3D reconstruction may become a new approach to accurately measure cervical cancer tumors. MRI-3D reconstruction showed larger cervical cancer maximum tumor diameters, which resulted in an upstaging of approximately $30 \%$ of patients. Cervical cancer with LNM was associated with a significantly larger tumor volume.

\section{Acknowledgments}

The authors thank VitaWorks ${ }^{\circledR}$ Technology Co., Ltd. (Shanghai, China) for providing technical and software support. Vitaworks ${ }^{\circledR}$ had no control of the data or information submitted for publication, which remained the sole responsibility of the authors. The authors thank Huimei Zhou, Peng Peng, Ying Zhang, Jinhui Wang, Ninghai Cheng, Mei Yu, Jiaxin Yang, Department of Obstetrics and Gynecology, Peking Union Medical College Hospital, for their careful diagnosis and treatment of enrolled patients. The authors also thank the staff of Department of Radiology, Peking Union Medical College Hospital, for their diligent imaging-related work of enrolled patients.

Funding: This study was supported by the Fundamental Research Funds of Central Public Welfare Scientific Institution of the Chinese Academy of Medical Science [2019PT320011].

\section{Footnote}

Reporting Checklist: The authors have completed the STARD reporting checklist. Available at https://dx.doi. org/10.21037/atm-21-2246

Data Sharing Statement: Available at https://dx.doi. org/10.21037/atm-21-2246

Conflicts of Interest: All authors have completed the ICMJE 
uniform disclosure form (available at https://dx.doi. org/10.21037/atm-21-2246). The authors have no conflicts of interest to declare.

Ethical Statement: The authors are accountable for all aspects of the work in ensuring that questions related to the accuracy or integrity of any part of the work are appropriately investigated and resolved. The study was conducted in accordance with the Declaration of Helsinki (as revised in 2013). Ethical approval was granted by the institutional review board of Peking Union Medical College Hospital (approval number: JS-2082). Written informed consent for participation was waived for this retrospective study.

Open Access Statement: This is an Open Access article distributed in accordance with the Creative Commons Attribution-NonCommercial-NoDerivs 4.0 International License (CC BY-NC-ND 4.0), which permits the noncommercial replication and distribution of the article with the strict proviso that no changes or edits are made and the original work is properly cited (including links to both the formal publication through the relevant DOI and the license). See: https://creativecommons.org/licenses/by-nc-nd/4.0/.

\section{References}

1. Cohen PA, Jhingran A, Oaknin A, et al. Cervical cancer. Lancet 2019;393:169-82.

2. Bhatla N, Aoki D, Sharma DN, et al. Cancer of the cervix uteri. Int J Gynaecol Obstet 2018;143 Suppl 2:22-36.

3. Kim HJ, Kim W. Method of tumor volume evaluation using magnetic resonance imaging for outcome prediction in cervical cancer treated with concurrent chemotherapy and radiotherapy. Radiat Oncol J 2012;30:70-7.

4. Lee G, Park JY, Kim DY, et al. Comparison of MRI, PET/ CT, and frozen biopsy in the evaluation of lymph node status before fertility-sparing radical trachelectomy in early stage cervical cancer. Eur J Cancer 2017;72:S92.

5. Joo YH, Hwang SH, Sun DI, et al. Relationships between Tumor Volume and Lymphatic Metastasis and Prognosis in Early Oral Tongue Cancer. Clin Exp Otorhinolaryngol 2013;6:243-8.

6. Durso TA, Carnell J, Turk TT, et al. Three-dimensional reconstruction volume: a novel method for volume measurement in kidney cancer. J Endourol 2014;28:745-50.

7. Le TN, Bao PT, Huynh HT. Fully automatic scheme for measuring liver volume in 3D MR images. Biomed Mater Eng 2015;26 Suppl 1:S1361-9.
8. Li P, Liu P, Chen C, et al. The 3D reconstructions of female pelvic autonomic nerves and their related organs based on MRI: a first step towards neuronavigation during nervesparing radical hysterectomy. Eur Radiol 2018;28:4561-9.

9. Takekuma M, Kasamatsu Y, Kado N, et al. The issues regarding postoperative adjuvant therapy and prognostic risk factors for patients with stage I-II cervical cancer: A review. J Obstet Gynaecol Res 2017;43:617-26.

10. Wang T, Gao T, Yang J, et al. Preoperative prediction of pelvic lymph nodes metastasis in early-stage cervical cancer using radiomics nomogram developed based on T2-weighted MRI and diffusion-weighted imaging. Eur J Radiol 2019;114:128-35.

11. Liu C, Ding J, Spuhler K, et al. Preoperative prediction of sentinel lymph node metastasis in breast cancer by radiomic signatures from dynamic contrast-enhanced MRI. J Magn Reson Imaging 2019;49:131-40.

12. Downey K, Jafar M, Attygalle AD, et al. Influencing surgical management in patients with carcinoma of the cervix using a T2- and ZOOM-diffusion-weighted endovaginal MRI technique. Br J Cancer 2013;109:615-22.

13. Wagenaar HC, Trimbos JB, Postema S, et al. Tumor diameter and volume assessed by magnetic resonance imaging in the prediction of outcome for invasive cervical cancer. Gynecol Oncol 2001;82:474-82.

14. Chen AC, Sung WH, Wang PH, et al. Correlation of three-dimensional tumor volumetry with cervical cancer prognostic parameters. Eur J Gynaecol Oncol 2002;23:401-4.

15. Fujii S, Iwata $\mathrm{N}$, Inoue $\mathrm{C}$, et al. Volume Measurement by Diffusion-Weighted Imaging in Cervical Cancer. Yonago Acta Med 2017;60:113-8.

16. Zhang J, Cao D, Yang J, et al. Tumor Volume Predicts High-Risk Patients and Guides Initial Chemoradiotherapy for Early Cervical Cancer. Front Oncol 2021;11:640846.

17. Chen XL, Chen GW, Xu GH, et al. Tumor Size at Magnetic Resonance Imaging Association With Lymph Node Metastasis and Lymphovascular Space Invasion in Resectable Cervical Cancer: A Multicenter Evaluation of Surgical Specimens. Int J Gynecol Cancer 2018;28:1545-52.

Cite this article as: Zhang J, Wang Y, Cao D, Shen K. MRIbased three-dimensional reconstruction for staging cervical cancer and predicting high-risk patients. Ann Transl Med 2021;9(18):1398. doi: 10.21037/atm-21-2246 